DEGUM-Mitteilungen

Deutsche Gesellschaft für Ultraschall in der Medizin

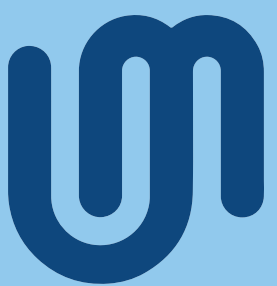

Online-Pressekonferenz der DEGUM: Ultraschall und Augenerkrankungen

\section{Wie Sonografie Erblindung verhindern kann}

Wer sein Augenlicht verliert, verliert in der Regel auch erheblich an der Teilhabe am gesellschaftlichen Leben und büßt viel an Lebensqualität ein. Bei drohendem Sehverlust muss deshalb schnell, effektiv und sicher gehandelt werden. Der Ultraschall ist eine schonende, strahlungsarme und vor allem vielfältig einsetzbare Methode. Wie sie bei unterschiedlichen Augenerkrankungen zum Einsatz kommt, haben Experten der DEGUM bei einer Pressekonferenz zum Thema „Ultraschall und Augenerkrankungen“ am 1. Dezember 2021 erläutert. Die Konferenz fand online statt.
Seit Anfang 2021 fordert die Strahlenschutzverordnung die Reduktion ionisierender Strahlung in der Augenmedizin. Denn gerade die Augenlinse gehört zu den strahlenempfindlichen Gewebestrukturen im menschlichen Körper. Sie nimmt ionisierende Strahlung auf, was krankhafte Veränderungen an der Linsenstruktur zur Folge haben kann. „Langzeituntersuchungen und epidemiologische Datenerhebungen über die Folgen von Strahlung fanden zwischenzeitlich heraus, dass wiederholte niedrige Strahlendosen ebenfalls einen strahleninduzierten Katarakt hervorrufen können“, sagte Professor Dr. med. Frank Tost, Leitender Oberarzt an der
Klinik und Poliklinik für Augenheilkunde der Universitätsmedizin Greifswald und Leiter der DEGUM-Sektion Ophthalmologie bei der Online-Pressekonferenz. Mit der Aktualisierung der Strahlenschutzverordnung wie auch der AWMF-Leitlinie (Reg.Nr. 039-93) zur radiologischen Diagnostik im Kopf-HalsBereich seien Vorkehrungen getroffen worden, die zur Patientensicherheit beitrügen, so Tost, und auch zu mehr Arbeitssicherheit für medizinisches Personal und Flugpersonal führten. Das zunehmende Bewusstsein der besonderen Strahlenempfindlichkeit der Augenlinse erfordere die Vermeidung jeder Strahlenbelastung der Augen bei Fragestel- 
lungen, für die andere, alternative bildgebende Untersuchungsverfahren zur Verfügung stünden.

Mithilfe des Ultraschalls kann die Strahlenbelastung oftmals vermieden oder zumindest reduziert werden. Als Beispiele nannte Tost Verletzungen des Auges und den Ausschluss oder Nachweis von Fremdkörpern. „Der Ultraschall ist geeignet, besonders kleine Fremdkörper zu erfassen und vor allem auch Material, das im CT/MRT nicht darstellbar ist, wie Brillenglassplitter, Kunststoffe, Pflanzenteile. Das MRT sollte bei unklaren Verletzungen nicht angewandt werden, da Metallfremdkörper dann großen zusätzlichen Schaden beim Betroffenen anrichten“, so der DEGUM-Augenexperte. Doch auch etwa bei plötzlicher einseitiger Augen-Innendruck-Erhöhung (Sekundärglaukom) lassen sich mit Ultraschall wichtige bildgebende Hinweise erfassen. Schon allein der Einsatz verschiedener Ultraschallsonden kann zur Strahlenbelastung führen. Aus augenärztlicher Sicht sei es besonders wichtig, Ultraschallsonden nach ihren speziellen Eigenschaften (Ultraschallfrequenz, Abbildungsgeometrie, Auflösungsvermögen und Eindringtiefe) optimal am Auge einzusetzen: Linearscanner mit hoher Frequenz seien besonders optimal am vorderen Auge (Hornhaut, Kammerwinkel, Augenlinse) anwendbar. Sektorscanner mit konkaver Abbildungsgeometrie seien hingegen besonders gut für das hintere Augensegment (Glaskörper, Netzhaut, Sehnerv) geeignet.

\section{Wie den Grauen Star \\ behandeln? Laser ohne}

\section{Mehrwert}

Auch in der Therapie von AugenlinsenErkrankungen - allen voran dem Grauen Star oder Katarakt - wird Ultraschall eingesetzt. Seit Jahrzehnten gilt die LinsenkernZertrümmerung mittels Ultraschalls als Gold-Standard in der Behandlung des Grauen Stars. Mit fortschreitendem Alter bleibt fast niemand von der Linsentrübung verschont, die die Sicht immer weiter beeinträchtigt und unbehandelt bis zur Blindheit führen kann. „Ab einem Alter von 65 bis 75 Jahren sind deutlich über $90 \%$ der Menschen von Linsentrübungen betroffen“, sagte PD Dr. Ulrich Fries, Chefarzt der Augenklinik an den Johanniter-Kliniken Bonn und Stellvertretender Leiter der DEGUM-Sektion Ophthalmologie, bei der Pressekonferenz. Bei rund jedem Zweiten sei die Linsentrübung so ausgeprägt, dass sie sich als Sehbeeinträchtigung bemerkbar mache. Entsprechend häufig werde der Graue Star operativ behandelt. „Mit jährlich rund 800000 Eingriffen ist die KataraktOperation die häufigste und sicherste Operation in Deutschland überhaupt", sagte Fries. Die getrübte Linse werde dabei entfernt und durch eine Kunstlinse ersetzt. Klassischerweise werde die Linse dabei im Auge mithilfe eines Ultraschallhandstücks zertrümmert, das durch einen kleinen seitlichen Schnitt zunächst in das Auge und dann in die Linsenkapsel eingeführt werde. Die Trümmerstücke würden abgesaugt, anschließend werde eine individuell angepasste Kunststofflinse in das im Auge verbleibende Kapselhäutchen eingebracht.

Seit einigen Jahren steht mit der sogenannten Laser-Phakoemulsifikation ein alternatives Verfahren zur Verfügung, bei dem die Linse mithilfe eines Femto-Sekunden-Lasers zerkleinert wird, der mit ultrakurzen Lichtpulsen arbeitet. „Die Schnitte im Auge werden dabei mit dem Laser vorbereitet, und auch die Linsenzertrümmerung findet per Laser statt“, erklärte Fries. Das klassische Saug-Spül-Verfahren werde jedoch weiterhin benötigt, zum Teil müsse auch per Ultraschall nachgearbeitet werden, weil der Laser sehr kapselnahe Linsenanteile nicht immer gefahrlos zerkleinern könne. „Hier kommt unter Umständen ein zusätzlicher Operationsschritt auf die Patienten zu“, so Fries. In der Hand erfahrener Operateure seien beide Verfahren sehr sicher und führten zum gleichen operativen Ergebnis, betonte der Experte. Der Lasereinsatz sei jedoch deutlich teurer - pro Auge entstehe ein Kostenplus von rund $1500 €$, das der Patient selbst tragen müsse. „Das Femto-Laser-Verfahren hat noch keine Gebührenordnungsziffer und kann somit nur privat abgerechnet werden“, so Fries. Angesichts des fehlenden Mehrwerts könne er nur davon abraten, den Laser dem Ultraschall-basierten Eingriff vorzuziehen. Dieser sei eine voll bezahlte Kassenleistung und habe sich millionenfach bewährt.

\section{Riesenzell-Arteriitis: Gefäßsonografie verhindert Erblindung}

Innerhalb weniger Tage das Augenlicht zu verlieren - dieser Albtraum kann für Patientinnen und Patienten, die an einer Riesenzell-Arteriitis erkrankt sind, Wirklichkeit werden. Die Riesenzell-Arteriitis zählt zu den entzündlich-rheumatischen Erkrankungen, bei denen sich das Immunsystem gegen körpereigene Strukturen wendet und zu Entzündungen führt. Während beim deutlich häufigeren und bekannteren Gelenkrheuma die Gelenke schmerzhaft entzündet sind, sind bei der Riesenzell-Arteriitis hauptsächlich die mittleren und großen Blutgefäße im Hals- und Schläfenbereich betroffen. „Die Wände dieser Blutgefäße schwellen durch das Entzündungsgeschehen stark an und der Gefäßinnenraum verengt sich, was bis zum völligen Gefäßverschluss führen kann“, erläuterte Professor Dr. med. Wolfgang Hartung, Leitender Oberarzt an der Klinik und Poliklinik für Rheumatologie und Klinische Immunologie am Asklepios-Klinikum Bad Abbach und Leiter des Arbeitskreises Bewegungsorgane der DEGUM, bei der Pressekonferenz. Weil auch die Augenarterien von diesen Veränderungen betroffen sind, wird in vielen Fällen auch die empfindliche Augennetzhaut nicht mehr ausreichend mit Blut versorgt. In der Folge können schwerwiegende Schäden entstehen, die bis zur Erblindung reichen.

Ein frühes und charakteristisches Symptom der Riesenzell-Arteriitis sind neu auftretende, meist beidseitige Schläfenkopfschmerzen. Auch Schmerzen beim Kauen sind häufig, denn auch die Kaumuskeln werden nur noch eingeschränkt mit Blut versorgt. „Bereits diese Symptome sollten als Warnsignale ernst genommen und sofort diagnostisch abgeklärt werden“, sagte Hartung. Bei 20 bis $50 \%$ der Patientinnen und Patienten träten außerdem bereits zum Zeitpunkt der Diagnose Sehstörungen auf, wie etwa unscharfes Sehen, Doppelbilder oder ein plötzlicher Sehverlust. „In diesem Fall muss sofort eine hochdosierte Kortisonbehandlung eingeleitet werden, um bleibende Schäden an den Augen zu verhindern“, so Hartung. Um die Verdachtsdiagnose einer Riesenzell-Arteriitis schnell und zuverlässig 
zu überprüfen, sei die Ultraschall-Untersuchung der Schläfenarterien unverzichtbar. Als rasch einsetzbares, sehr aussagekräftiges und nichtinvasives Verfahren gelte sie mittlerweile auch international als Goldstandard. Falls zur zusätzlichen Absicherung der Diagnose noch eine Gewebeprobe entnommen werden müsse, könne unter Ultraschall-Führung zudem ein geeigneter Gefäßabschnitt ausgewählt werden. „Mit Ultraschall lassen sich die Orte der Gefäßentzündung genau identifizieren und stumme Zonen, also Gefäßabschnitte ohne Entzündung, können dann gemieden werden“, ergänzte Professor Tost. Auch in der Verlaufskontrolle habe die Ultraschall-Untersuchung ihren festen Platz. Mit ihr lasse sich die Entwicklung des Entzündungsgeschehens objektiv kontrollieren und die Therapie bei Bedarf anpassen.

\section{Endokrine Orbitopathie: Ultraschall als wichtige Entscheidungshilfe}

Auch bei hervortretenden Augen, die eine Folge bestimmter Schilddrüsen-Erkrankungen sein können, wird Ultraschall im
Rahmen der Behandlung erfolgreich eingesetzt. Hierzu zählt die Endokrine Orbitopathie, eine entzündliche Erkrankung der Augenhöhle. Die Betroffenen leiden unter Schmerzen, Rötung sowie Schwellung der Augen. Außerdem ist die Wahrnehmung von Doppelbildern nicht selten. Typisches äußerlich wahrnehmbares Merkmal der Erkrankung ist das Hervortreten der Augen (Exophthalmus) und ein starrer Blick. „Bei Beteiligung des Sehnervs kann es zu ernsthaftem Sehverlust bis hin zur Erblindung kommen “, erläuterte PD Dr. Ulrich Fries bei der Pressekonferenz.

Der Ultraschall-Experte legte bei der Pressekonferenz dar, wie sowohl der Sehnerv als auch die Augenmuskeln, die Tränendrüse und das Fettgewebe sonografisch untersucht und die Ergebnisse dann zur weiteren Therapieplanung und Therapiekontrolle eingesetzt werden können. Fragestellungen wie, welche Struktur betroffen ist, ob eine Optikuskompression besteht oder ob ein Ödem oder eine Fibrose vorliegen, könnten mithilfe des Ultraschalls beantwortet werden. „Mittels Ultraschalls können die vorderen zwei Drittel der Augenmuskeln untersucht und beurteilt werden“, so Fries weiter. Die Experten unterscheiden dabei zwischen Augenmuskeln mit normaler Echogenität und solchen mit erniedrigter Echogenität - letzteres deute auf Muskeln mit Entzündungszeichen hin. Eine angehobene Echogenität wiederum sei ein Zeichen der Vernarbung und damit der Abheilung des entzündlichen Geschehens. Jeder Augenmuskel könne einzeln betrachtet werden, in der dynamischen Sonografie auch bei Kommandobewegungen. Die Dicke des Muskelbauches könne etwa im mittleren Bereich gemessen werden. „Die endokrine Orbitopathie führt insbesondere zu Muskelverbreiterungen in der Orbitaspitze, welche sich der Ultraschall-Untersuchung entzieht“, erklärte Fries. Die Gefährdung für das Augenlicht ließe sich eindeutig durch die Messung des Sehnervendurchmessers abschätzen.

An der Pressekonferenz nahmen rund 35 Journalisten teil. Das Interesse war groß, es wurden viele Nachfragen gestellt. Wer die Pressekonferenz verpasst hat, kann sie jederzeit nachträglich anschauen: Den Link gibt es auf www.degum.de. 